\title{
Unterschiede im Leistungsverhalten von Gesunden zwischen Fahrrad- und Laufbandergometrie
}

\author{
A. Schuh, G. Baranek, V. Entleutner, E. Senn \\ Institut für Medizinische Balneologie und Klimatologie der Universität München (Vorstand: Prof. Dr. med. E. Senn)
}

\section{Research comparing the use of bicycle and treadmill ergometry in performance tests with healthy persons}

Objective: In Terms of endurance training (terrain cure), treadmill ergometry was used as a test method for recording the success of the cure, straining the same muscles which are under strain during the training.

However, due to the early subjective exhaustion of the patients, lactic acid values above the aerobic/anaerobic threshold will not be reached very often, the patients will rarely reach submaximal load.

Subject: This study searches for the reason of this lack of metabolic submaximal load of the patients and determines the differences of load between bicycle and treadmill ergometry.

Design: 27 healthy persons were put under submaximal load on bicycle and treadmill ergometers. The load was increased in $0.33 \mathrm{~W} / \mathrm{kg}$ steps. Most important measuring parameters were heart rate and blood lactate concentration.

Results: During treadmill ergometry, heart rate and myocardial oxygen requirement to reach the same watt-level were significantly higher $(1.0 \mathrm{~W} / \mathrm{kg}: \mathrm{p} \leq 0.001$; $1.33 \mathrm{~W} / \mathrm{kg}: \mathrm{p}-0.01 ; 2.0 \mathrm{~W} / \mathrm{kg}$ : n.s.; recreation: $\mathrm{p} \leq 0.001$ ) than on the bicycle. On the other hand with determined heart rate $(130 / \mathrm{min})$ and on the same maximal watt-level a significantly $(\mathrm{p} \leq 0.001)$ lower increase in lactate by $0.7 \mathrm{mmol} / 1$ and $1.0 \mathrm{mmol} / \mathrm{l}$, respectively than on the bicycle ergometer was found.

Conclusion: The results show stronger cardiac exhaustion on the treadmill ergometer. But the aerobic/anaerobic threshold is reached on the treadmill later than on the bicycle.

\section{Key words}

Bicycle ergometry - treadmill ergometry cardiac exhaust - aerob/anaerob threshold

\section{Kurzfassung}

Problemstellung: Zur Überprüfung des Ausdauertrainingseffektes während einer Terrainkur ist die Laufbandergometrie der Belastung auf dem Fahrrad vorzuziehen, da hierbei dieselben Muskeln beansprucht werden wie während des Trainings. Allerdings gelingt es wegen der frühzeitigen subjektiven Erschöpfung der Kurpatienten nur selten, Milchsäurewerte oberhalb der aeroben/anaeroben Schwelle zu erzielen.

Gegenstand: Die vorliegende Studie mit Gesunden befaßt sich mit den Fragen, warum sich Kurpatienten auf dem Laufband metabolisch betrachtet nicht genügend ausbelasten lassen, und welches die entscheidenden Unterschiede in der Art der Belastung zwischen Laufband- und Fahrradergometer sind.

Versuchsplan: 27 gesunde Testpersonen wurden auf dem Fahrrad- und Laufbandergometer submaximal belastet. Die Belastung wurde nach einem standardisierten Verfahren in $0,33 \mathrm{~W} / \mathrm{kg}$-Schritten gesteigert. Wichtigste Meßparameter waren Herzfrequenz und Milchsäurespiegel.

Ergebnisse: Während der Laufbandergometrie lagen Herzfrequenz und Sauerstoffbedarf des Myokards zum Erreichen der gleichen Wattstufe signifikant höher $(1,0$ $\mathrm{W} / \mathrm{kg}$ : $\mathrm{p} \leq 0,001 ; 1,33 \mathrm{~W} / \mathrm{kg}: \mathrm{p} \leq 0,01 ; 2,0 \mathrm{~W} / \mathrm{kg}$ : n. s.; Erholung: $p \leq 0,001$ ) als auf dem Fahrrad. Dagegen zeigte sich bei gegebener Herzfrequenz von $130 / \mathrm{min}$ und bei derselben höchsten erreichten Wattstufe auf dem Laufband ein um 0,7 $\mathrm{mmol} / \mathrm{l}$ bzw. $1,0 \mathrm{mmol} / 1$ signifikant $(\mathrm{p} \leq 0,01)$ niedrigerer Laktatanstieg als auf dem Fahrradergometer.

Schlußfolgerung: Die Ergebnisse zeigten somit eine stärkere kardiale Ausschöpfung auf dem Laufbandergometer. Die aeroben/anaerobe Schwelle wird dagegen auf dem Laufband später als auf dem Fahrrad erreicht.

\section{Schlüsselwörter}

Fahrradergometrie - Laufbandergometrie kardiale Anforderungen - metabolische Auslastung 


\begin{tabular}{|c|c|c|c|c|c|c|c|c|c|c|}
\hline $\mathrm{W} / \mathrm{kg}$ & 4 & 5 & 6 & 7 & $\begin{array}{c}\mathrm{km} / \mathrm{h} \\
8\end{array}$ & 9 & 10 & 11 & 12 & \\
\hline 1 & 9 & 7,2 & 6 & 5,2 & 4,5 & 4 & 3,6 & 3,25 & 3 & S \\
\hline 2 & 18 & 14,4 & 12 & 10,4 & 9,0 & 8 & 7,2 & 6,50 & 6 & $\frac{D}{0}$ \\
\hline 3 & 27 & 21,6 & 18 & 15,6 & 13,5 & 12 & 10,8 & 9,75 & 9 & \\
\hline 4 & 36 & 28,6 & 24 & 20,8 & 18,0 & 16 & 14,4 & 13,00 & 12 & \\
\hline 5 & 45 & 36,0 & 26 & 22,5 & 20,0 & 18 & 16,5 & 15,00 & 15 & \\
\hline
\end{tabular}

konstante Gehgeschwindigkeit, steigende Laufbandneigung
Tab. 1 Die zu jeder Laufbandgeschwindigkeit von $4-12 \mathrm{~km} / \mathrm{h}$ zugehörige Laufbandneigung, um die Belastung von $1 \mathrm{~W} / \mathrm{kg}$ bis $5 \mathrm{~W} / \mathrm{kg}$ Körpergewicht zu ermöglichen. Angaben einer $W / \mathrm{kg}$ körpergewichtsbezogenen Variation der Gehgeschwindigkeit und des Steigerungswinkels (Nowacki 1980).

\section{Einleitung}

Im Rahmen der Klimatherapieforschung steht das kurmäßig dosierte Ausdauertraining (Terrainkur) zur Zeit im Vordergrund; deshalb war es notwendig, ein Testverfahren zur Erfassung des Kureffektes einzusetzen, bei welchem dieselben Muskeln wie während des Trainings beansprucht werden. Zur Überprüfung des Trainingseffektes ist aus dieser Sicht die Laufbandergometrie der Belastung auf dem Fahrrad vorzuziehen.

Bei den Laufbanduntersuchungen an Kurpatienten fällt allerdings immer wieder auf, daß es wegen der frühzeitigen subjektiven Erschöpfung der Patienten nur selten gelingt, Milchsäurewerte an der aeroben/anaeroben Schwelle zu erzielen, d.h., die Patienten auf dem Laufband metabolisch auszubelasten: In einer kürzlich publizierten Studie ( $\mathrm{Abkai}$ 1992) zum Beispiel, erreichten untrainierte Kurpatienten $(\mathrm{n}=$ 75, Durchschnittsalter 50 Jahre) auf dem Laufband bereits bei einer Leistung von 1,0 W/kg Körpergewicht eine Herzfrequenz von $150 / \mathrm{min}$; sie brachen in der Regel die Belastung bei 1,33 W/kg Körpergewicht und einer Herzfrequenz von $170 / \mathrm{min}$ ab, obwohl die Milchsäurewerte dabei erst auf etwa $3,5 \mathrm{mmol} / \mathrm{l}$ angestiegen waren. Der Abbruch erfolgte zumeist aufgrund eines subjektiven Erschöpfungsgefühls, das mit hohen Herzfrequenzen einherging

Die Ergebnisse einer früheren Untersuchung (Schuh 1989) zeigten dagegen, daß ein von Alter, Indikation und Trainingszustand her vergleichbares Patientenkollektiv ( $\mathrm{n}=$ 98) auf dem Fahrradergometer bei $1,0 \mathrm{~W} / \mathrm{kg}$ Körpergewicht lediglich eine Herzfrequenz von $125 / \mathrm{min}$ und bei $1,33 \mathrm{~W} / \mathrm{kg}$ Körpergewicht von $140 / \mathrm{min}$ erreichte. Die Belastung wurde erst bei einer Höhe von $2,0 \mathrm{~W} / \mathrm{kg}$ Körpergewicht mit einer Herzfrequenz von 160/min abgebrochen. Die Laktatwerte lagen bei der mittleren Belastung von 1,33 W/kg Körpergewicht bereits auf einer Höhe von $4,5 \mathrm{mmol} / \mathrm{l}$, beim Abbruch von 6,1 $\mathrm{mmol} / \mathrm{l}$.

Die qualitativen Unterschiede in der Belastung von Herz und Kreislauf sind bereits mehrfach bearbeitet worden (Hollmann et al. 1987); die Ergebnisse bezüglich des Herzfrequenzverhaltens weichen allerdings bei einigen Autoren erheblich voneinander ab (Worms et al. 1984, Nowacki 1980).

Für die nachfolgende Untersuchung an Gesunden stehen somit zwei Fragen im Mittelpunkt:

1. Warum lassen sich Kurpatienten auf dem Laufband metabolisch betrachtet nicht genügend ausbelasten?

2. Welches sind die entscheidenden Unterschiede in der Art der Belastung zwischen Laufband- und Fahrradergometer?

\section{Methodik}

27 gesunde Probanden, im Alter von 20-52 Jahren (Durchschnittsalter 26 Jahre), 7 Frauen und 20 Männer, nahmen an den Untersuchungen teil.

Die Probanden wurden randomisiert sowohl auf dem Fahrrad als auch auf dem Laufband in ansteigenden Stufen von 3 Minuten belastet. Um auf dem Laufband das zu tragende Körpergewicht zu berücksichtigen, wurde das Dosierungsverfahren von Nowacki (1989) gewählt, welches die Leistung auf W/kg Körpergewicht bezieht (Tab. 1): Bei konstanter Gehgeschwindigkeit von $4 \mathrm{~km} /$ Stunde wurde die Steigerung des Laufbandes - ausgehend von $3 \%$ - so verändert $(6 \%, 9 \%$, $12 \%, 15 \%, 18 \%$ etc.), daß die Belastung in $1 / 3 \mathrm{~W} / \mathrm{kg}-$ Körpergewichtsschritten zunahm. Die Fahrradbelastung wurde bei 50 Umdrehungen pro Minute ebenfalls um jeweils $1 / 3 \mathrm{~W} / \mathrm{kg}$ Körpergewicht gesteigert.

Die Herzfrequenz wurde während der Ergometrie kontinuierlich registriert; der direkte Vergleich der Meßergebnisse zwischen Laufband- und Fahrradergometrie wurde für Herzfrequenz, Blutdruck und das Puls-Blutdruck-Produkt in Ruhe $(\mathrm{n}=27)$, bei $1,0 \mathrm{~W} / \mathrm{kg}(\mathrm{n}=27), 1,33 \mathrm{~W} / \mathrm{kg}(\mathrm{n}=27)$ und $2,0 \mathrm{~W} / \mathrm{kg}$ Körpergewicht $(\mathrm{n}=21)$ vorgenommen. Die Milchsäurewerte im arteriellen Blut wurden in Ruhe, bei Erreichen der Herzfrequenz von $130 / \mathrm{min}$ sowie 2 Minuten nach Abbruch bzw. Ende der Belastung bestimmt (jeweils $n=27$ ). Als , individuell höchste" Belastungsstufe galt dabei jene selbe Wattstufe, die von den Teilnehmern sowohl auf dem Laufband als auch auf dem Fahrrad erreicht wurde.

Der Abbruch erfolgte aufgrund des subjektiven Gefühls, ausbelastet zu sein: 6 Personen konnten in beiden Tests bis 2,66 W/kg Körpergewicht belastet werden; die übrigen 21 brachen bereits bei 1,66 bzw. 2,0 W/kg Körpergewicht ab. Bei 13 Probanden lag dabei die subjektive Erschöpfung nur bei dem ersten der beiden Ergometrieformen vor, beim anderen Belastungstest ließen sie sich bis $2,33 \mathrm{~W} / \mathrm{kg}$ bzw. 2,66 W/kg Körpergewicht belasten. In diesen Fällen kamen zusätzlich zu den Messungen bei 2,0 W/kg Körpergewicht (,,individuell höchste“" Belastungsstufe bei Laufband und Fahrrad) noch HF-, RR- und Laktatwerte am Ende der länger durchgehaltenen Ergometrie dazu. Entsprechend konnten die Erholungswerte nur von den 14 Probanden ausgewertet werden, die den Test bei derselben Belastungsstufe abbrachen.

Die statistische Auswertung wurde mit dem Student t-Test für paarige Verbindungen vorgenommen. Für die Irrtumswahrscheinlichkeit wurde das Signifikanzniveau von $1 \%\left(\mathrm{p} \leq 0,01^{* *}\right)$ und $0,1 \%\left(\mathrm{p} \leq 0,001^{* * *}\right)$ angewendet. 


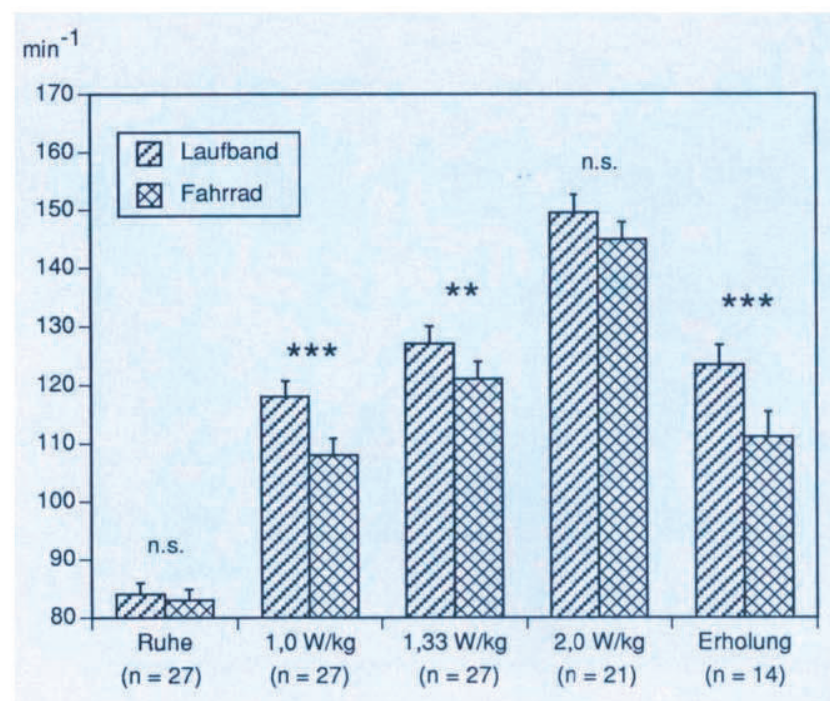

Abb. 1 Mittlere Herzfrequenz der gesunden Probanden auf Laufband und Fahrrad; in Ruhe, bei Belastung von $1,0 \mathrm{~W} / \mathrm{kg}$, $1,33 \mathrm{~W} / \mathrm{kg}$ (jeweils $\mathrm{n}=27$ ) und $2,0 \mathrm{~W} / \mathrm{kg}$ Körpergewicht $(\mathrm{n}=21)$ sowie drei Minuten nach Beendigung der Belastung (Erholung, $n=14)$.

\section{Ergebnisse}

Bei gleichem, nicht signifikant verschiedenem Ruhepuls liegt die durchschnittliche Herzfrequenz aller Probanden (Abb. 1) bei derselben Belastung von 1,0 W/kg Körpergewicht auf dem Laufband um $11 /$ min signifikant ( $\leq \leq 0,001)$ höher als auf dem Fahrrad. Bei einer Belastung von $1,33 \mathrm{~W} / \mathrm{kg}$ Körpergewicht erreicht die Herzfrequenz auf dem Laufband $127 / \mathrm{min}$, während auf dem Fahrrad nur $120 / \mathrm{min}$ gemessen werden. Auch dieser Unterschied von $7 / \mathrm{min}$ ist signifikant ( $\mathrm{p} \leq$ $0,01)$. Bei weiterem Anstieg der Belastung werden die Pulsdifferenzen kleiner; bei 2,0 W/ kg Körpergewicht steigt die Herzfrequenz auf dem Laufband auf $150 / \mathrm{min}$, auf dem Fahrrad werden 145/min erreicht (n.s.). Die Erholungswerte liegen nach der Fahrradergometrie signifikant niedriger als nach Laufbandbelastung: Während zwei Minuten nach Abbruch des "Laufens" der durchschnittliche Puls noch bei $123 / \mathrm{min}$ liegt, haben dieselben Probanden zur gleichen Zeit nach dem Ende der Fahrradergometrie nur noch $111 / \mathrm{min}(\mathrm{p} \leq 0,001)$.

Der Anstieg der Herzfrequenz (Abb. 2) verläuft am Anfang der Ergometrie bei gleichmäßig ansteigender Belastung bis $1,0 \mathrm{~W} / \mathrm{kg}$ Körpergewicht auf dem Laufband signifikant ( $\mathrm{p} \leq 0,001)$ steiler als auf dem Fahrrad; die Herzfrequenz nimmt schneller zu (Steigerung der Geraden zwischen den Variablen HF und $\mathrm{W} / \mathrm{kg}$ Körpergewicht bei Laufbandergometrie $63 \%$, bei Fahrradergometrie 44\%). Dieses Anstiegsverhalten ändert sich im weiteren Verlauf: Bei Belastungszunahme von 1,0 über 1,33 auf 2,0 W/ kg Körpergewicht nimmt die Herzfrequenz bei der Fahrradergometrie stärker zu ( $80 \%)$ als auf dem Laufband (67\%) (bei 1,33 W/kg: p $\leq 0,01$ ). Obwohl sich die Differenzen zunehmend verringern, bleibt die Herzfrequenz bei Belastungen bis $2,0 \mathrm{~W} / \mathrm{kg}$ Körpergewicht auf dem Fahrrad niedriger als auf dem Laufband (n. s.).

Der systolische Blutdruck unterscheidet sich praktisch nicht: Er liegt während der Belastungsstufen bis 1,33

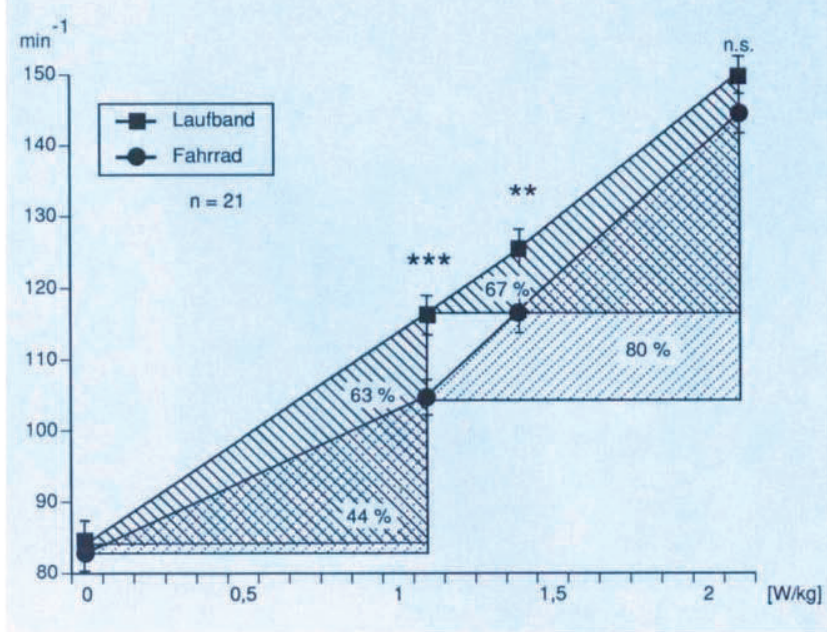

Abb. 2 Verlauf der mittleren Herzfrequenz der gesunden Probanden ( $\mathrm{n}=21$ ) auf Laufband und Fahrrad; in Ruhe, bei Belastung von $1,0 \mathrm{~W} / \mathrm{kg}, 1,33 \mathrm{~W} / \mathrm{kg}$ und $2,0 \mathrm{~W} / \mathrm{kg}$ Körpergewicht sowie drei Minuten nach Beendigung der Belastung (Erholung). Angabe der Steigerung der Geraden durch die Variablen HF und W/kg zwischen 0 und $1,0 \mathrm{~W} / \mathrm{kg}$ sowie 1,0 und $2,0 \mathrm{~W} / \mathrm{kg}$ Körpergewicht.

W/kg Körpergewicht auf dem Laufband etwas höher als auf dem Fahrrad (n.s.); bei höchster Belastung ist der RRs auf dem Fahrrad höher (n.s.).

Der myokardiale Sauerstoffverbrauch (Produkt von Herzfrequenz und systolischem Blutdruck, Abb. 3) ist, ausgehend von gleichen Ruhewerten, bei 1,0 (p $\leq 0,001)$ und 1,33 $\mathrm{W} / \mathrm{kg}$ Körpergewicht $(\mathrm{p} \leq 0,01)$ auf dem Laufband signifikant größer als auf dem Fahrrad. Bei der Belastung von $2,0 \mathrm{~W} / \mathrm{kg}$ Körpergewicht können allerdings keine Unterschiede mehr festgestellt werden. Die Erholungswerte sind nach Belastung auf dem Fahrrad niedriger als nach der Laufbandergometrie $(\mathrm{p} \leq 0,001)$.

Die beiden Belastungsarten unterscheiden sich auch in den Laktatwerten (Abb. 4): Bei der gegebenen Herzfrequenz von $130 / \mathrm{min}$ weisen die Probanden auf dem Laufband bei vergleichbaren Ruhewerten - noch keinen Anstieg der Milchsäurewerte auf, während auf dem Fahrrad bereits eine signifikante Zunahme um $0,7 \mathrm{mmol} / 1$ von 1,2 auf $1,0 \mathrm{mmol} / 1$ $\mathrm{zu}$ verzeichnen ist $(\mathrm{p} \leq 0,01)$. Die Laktatwerte der ,individuell höchsten" Belastungsstufe, welche von allen Teilnehmern auf dem Fahrrad als auch auf dem Laufband erreicht wurde, stiegen auf dem Laufband, ausgehend vom Ruhewert von $1,2 \mathrm{mmol} / \mathrm{kg}$, um $1,1 \mathrm{mmol} / 1$ auf $2,3 \mathrm{mmol} / 1$ an, während die Werte auf dem Fahrrad bei gleicher Belastung um 2,1 mmol/l auf 3,3 mmol/1 zunahmen. Die Laktatdifferenz von $1 \mathrm{mmol} / 1$ zwischen beiden Belastungsarten ist signifikant $(\mathrm{p} \leq 0,01)$.

Die Herzfrequenz 130/min wurde von den gesunden Probanden auf dem Laufband bereits bei einer Belastung von im Mittel 1,6 W/kg Körpergewicht, auf dem Fahrrad dagegen erst bei $1,9 \mathrm{~W} / \mathrm{kg}$ Körpergewicht erreicht $(\mathrm{p} \leq 0,01)$.

Die Leistungsfähigkeit der Probanden ist auf dem Laufband durchschnittlich höher als auf dem Fahrrad: Auf dem Laufband wurden - über die ,individuell höchste“ Bela- 


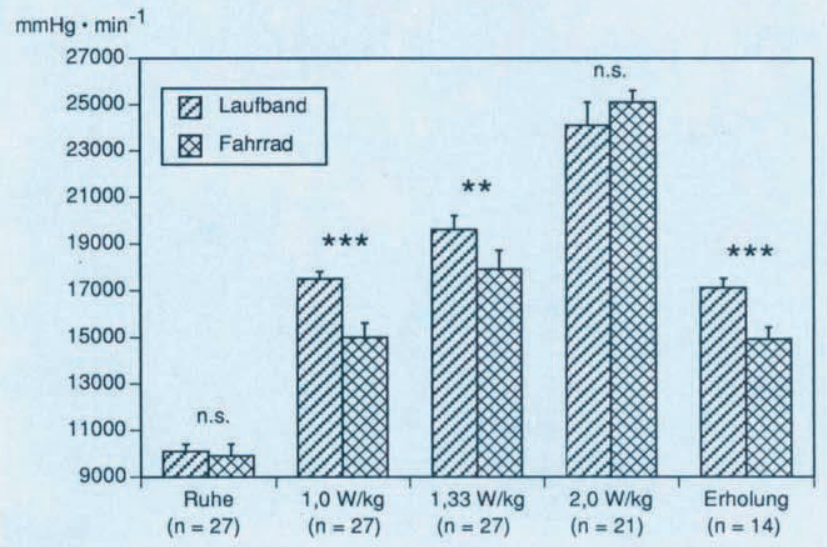

Abb. 3 Mittleres Produkt von Herzfrequenz und systolischem Blutdruck der gesunden Probanden auf Laufband und Fahrrad; in Ruhe, bei Belastung von $1,0 \mathrm{~W} / \mathrm{kg}, 1,33 \mathrm{~W} / \mathrm{kg}$ (jeweils $\mathrm{n}=27$ ) und $2,0 \mathrm{~W} / \mathrm{kg}$ Körpergewicht $(\mathrm{n}=21)$ sowie drei Minuten nach Beendigung der Belastung (Erholung, $n=14$ ).

stungsstufe hinaus - maximal 2,66 W/ kg Körpergewicht, auf dem Fahrrad von denselben Personen dagegen nur $2,33 \mathrm{~W} / \mathrm{kg}$ Körpergewicht erreicht $(n=13, p \leq 0,001)$.

\section{Diskussion}

Die Ergebnisse, die an gesunden Probanden verschiedenen Alters erarbeitet wurden, belegen durchwegs niedrigere Laktatspiegel bei Belastung auf dem Laufband gegenüber dem Fahrrad, wenn die Blut-Laktat-Konzentrationen auf die aktuelle Herzfrequenz bezogen werden. $\mathrm{Zu}$ demselben Ergebnis kommt auch eine Studie von Hollmann et al. (1987), bei welcher Sportstudenten einen Laktatspiegel von $4 \mathrm{mmol} / 1$ auf dem Fahrrad bereits bei einer Herzfrequenz von $153 / \mathrm{min}$, auf dem Laufband dagegen erst bei $173 /$ min erreichten.

Dieselben Autoren vermochten aufzuzeigen, daß die Laktatspiegel während der Laufbandergometrie ebenfalls niedriger sind als während der Fahrradergometrie, wenn diese auf die Höhe der Sauerstoffaufnahme bezogen werden.

Bei vergleichbaren Belastungsstufen sind auf dem Laufband nicht nur die Herzfrequenzen, sondern auch der Sauerstoffbedarf des Myokards (Produkt aus Herzfrequenz und systolischem Blutdruck) höher als auf dem Fahrrad.

Das Erreichen der aeroben/anaeroben Laktatschwelle stellt somit während der Laufbandergometrie höhere Anforderungen an das Herz-Kreislauf-System (Hollmann et al. 1987): Die Laktatschwelle wird erst bei einer deutlich höheren Leistung erreicht. Bei Laufbandbelastungen werden aufgrund der größeren beteiligten Muskelmasse höhere Frequenzen erreicht (Rost et al. 1982); während einer Fahrradergometrie kommt es zu einer auf die Körperleistung bezogene, früher einsetzende Übersäuerung des Kreislaufs.

Lehmann et al. (1983) sowie Pluto et al. (1987) wiesen auf dem Laufband geringere Katecholaminspiegel nach, wenn diese mit einer entsprechenden Leistung auf dem Fahrrad

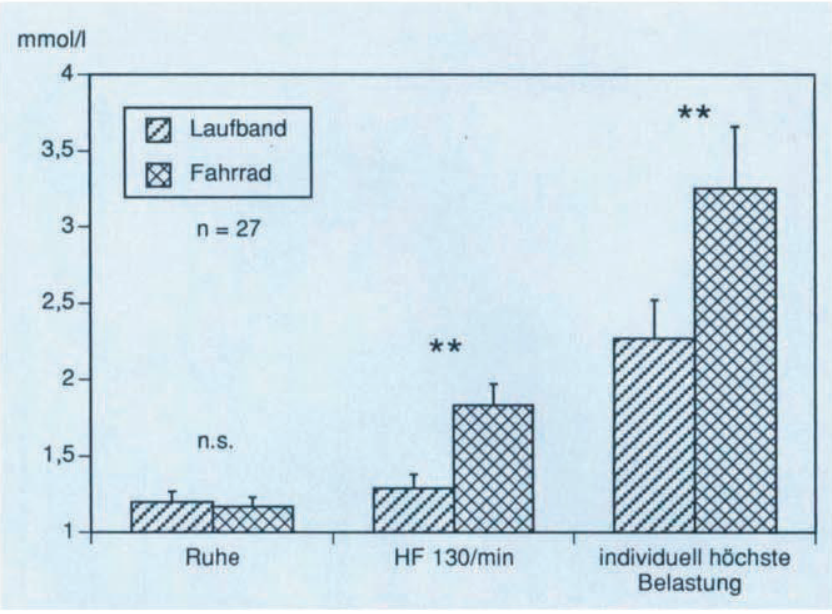

Abb. 4 Mittlerer Laktatspiegel der gesunden Probanden ( $n=27$ ) auf Laufband und Fahrrad; in Ruhe, bei gegebener Herzfrequenz von $130 / \mathrm{min}$ sowie bei derselben „individuell höchsten" Belastungsstufe.

verglichen wurden. Diese leistungsbezogene geringere Katecholaminfreisetzung geht mit einer reduzierten sogenannten Peitschenwirkung auf das kardiopulmonale System einher und wirkt sich dementsprechend positiv auf das Leistungsverhalten der Probanden aus.

Worms et al. (1984) dokumentierten darüber hinaus auf dem Laufband einen niedrigeren, auf die Herzfrequenz bezogenen, systolischen Blutdruck; dieser Zusammenhang konnte allerdings nicht statistisch signifikant wiederholt werden.

Die im Durchschnitt erreichbare, individuelle maximale Leistungsfähigkeit, ausgedrückt in W/kg Körpergewicht, ist unter Laufbandbedingungen höher als auf dem Fahrrad, weil beim „Laufen“ ganz offensichtlich eine größere Muskelmasse tätig wird. Je stärker sich die geforderte Leistung auf eine beschränkte Muskelmasse begrenzt, desto schneller müssen anaerob arbeitende, energieliefernde Stoffwechselwege eingesetzt werden.

Zu beachten gilt, daß während des Gehens auf dem Laufband gegenüber dem Fahrradfahren eine höhere innere, statische, sogenannte physiologische Arbeit geleistet werden muß, bevor eine Arbeitsleistung nach außen wirksam werden kann.

Während Laufbandbelastungen wird - bezogen auf entsprechende Laktatspiegel - das Herz-Kreislauf-System umfassender und intensiver beansprucht als während der Fahrradergometrie. Wenn es dagegen um das schnellere Erreichen eines Laktatspiegels im Übergangsbereich zwischen aerober und anaerober metabolischer Belastung geht, eignet sich die Fahrradergometrie besser als die Laufbandergometrie.

Bei der Testung bzw. beim Training von Patienten ist allerdings deren reduzierte Herz-Kreislauf-Belastbarkeit zu beachten, so daß in gewissen Fällen die schonendere Belastung auf dem Fahrrad dem Laufband vorzuziehen ist. 


\section{Literatur}

Abkai, L.: Freudenstädter Klimakur - Therapiekonzept zur Behandlung von Patienten mit Funktionsstörungen des Herz-Kreislauf-Systems ohne Organbefund. Med. Dissertation, München 1992

Hollmann, H., P. Schürch, H. Heck, H. Liesen, A. Mader, R. Rost, W. Hollmann: Kardiopulmonale Reaktionen und aerob-anaerobe Schwelle bei verschiedenen Belastungsformen. Dtsch. Z. Sportmed. 38 (1987) $144-156$

Lehmann, M., H. Dickhuth, K. Wybitul, A. Berg, G. Huber, J. Keul: Unterschiede der aeroben Ausbelastung, der freien Plasmakatecholamine und energieliefernden Substrate während Fahrrad-, Laufband und Gehbandergometrie. Dtsch. Z. Sportmed. 6 (1983) $188-194$

Nowacki, P. E.: Neue Aspekte der körpergewichtsbezogenen Fahrradund Laufbandergometrie für den Leistungs-, Breiten- und Rehabilitationssport. In: Kindermann, W. (Hrsg.): Sportmedizin für Breiten- und Leistungssport

Pluto, R., S. Cruze, T. Hotz, P. Mandel, M. Weiß, H. Weicker: Stoffwechselprozesse und venöse Katecholamine unter Ergometerbelastungen. Dtsch. Z. Sportmed. 38 (1987) 322-332

Rost R., W. Hollmann: Belastungsuntersuchungen in der Praxis. Thieme, Stuttgart (1982) 67

Worms, F, G. Kozariszczuk, K. Hunger: Blutdruckverhalten auf dem Laufband im Vergleich mit der Fahrradergometrie bei Normo- und Hypertonikern. Dt. Gesundh.-Wesen 39 (1984) 1779-1784

Schuh, A.: Das Krankheitsbild des Trainingsmangels und seine Behandlung durch Klimatherapie in Form einer Terrainkur unter kühlen Bedingungen. Habilitationsschrift, Medizinische Fakultät der Universität München 1989
Priv.-Doz. Dr. Dr. med. habil. Angela Schuh

Institut für Medizinische Balneologie und Klimatologie der Universität München

Marchioninistr. 17

8000 München 70 